\title{
A Low-Cost and Accurate Interface for Four-Electrode Conductivity Sensors
}

\author{
Xiujun Li, Senior Member, IEEE, and Gerard C. M. Meijer, Senior Member, IEEE
}

\begin{abstract}
This paper presents a low-cost and accurate interface for four-electrode conductivity sensors. The interface mainly consists of an analog front-end, a multiplexer, and a voltage-to-time converter. The analog front-end is used to provide a controlled excitation voltage for the sensor and to convert the sensor signal (conductance) into a voltage signal. The voltage-to-time period converter acts as an asynchronous converter for the sensor signals (voltage), which employs a relaxation oscillator and outputs a period-modulated signal. Experimental results over a conductance range of $0.1 \mu \mathrm{S}$ to $20 \mathrm{mS}$ show a random error of $1.6 \times 10^{-5}$ and a systematic error of $6.6 \times 10^{-5}$ for a measurement time of 110 ms.
\end{abstract}

Index Terms-Conductivity measurement, oscillators, sensors.

\section{INTRODUCTION}

C ONDUCTIVITY sensors are required in many application fields, including medical and biomedical fields, process chemistry, environment monitoring, agriculture and food production, etc. [1]-[4]. The accuracy and resolution of conductance measurements depend on chemical, physical, and electrical nonidealities. Regarding the chemical and physical effects, the main nonidealities concern:

- contamination of the electrode surface of the sensor;

- electrochemical effects;

- temperature dependence.

Considering the electrical effects, the main nonidealities concern:

- series impedances caused by the wires and cables, used to connect the conductivity sensor to the sensor electronics,

- effects of the dc drift and the influence of parasitic Seebeck voltages;

- effects of offset, $1 / f$ noise, and low-frequency interference;

- effects of multiplicative and additive errors of the processing circuit.

To overcome those nonidealities, in this paper a low-cost and accurate interface for four-electrode conductivity sensors is proposed, in which many advanced techniques are applied. These techniques include four-wire measurement, ac square-wave excitations, chopping [6], and autocalibration.

Manuscript received January 18, 2004; revised March 8, 2005. This work was supported by STW, the Dutch Technology Foundation, The Netherlands, under Project DMR 5294.

The authors are with the Faculty of Information Technology and Systems, Delft University of Technology, 2628 CD Delft, The Netherlands (e-mail: X.Li@ITS.tudelft.nl).

Digital Object Identifier 10.1109/TIM.2005.858130

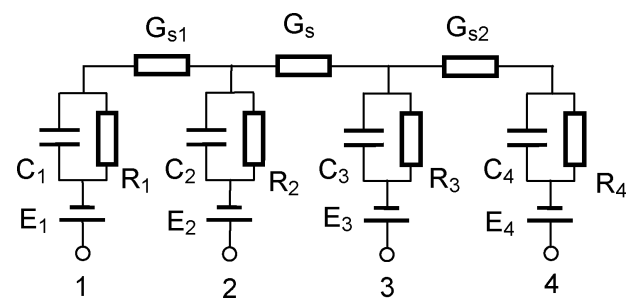

Fig. 1. A simple electrical model of the four-electrode conductivity sensor.

\section{MEASUREMENT PRINCIPLE}

\section{A. Model and Measurement of the Conductivity Sensor}

Fig. 1 shows a simple electrical model of a four-electrode conductivity sensor. In this model, the symbol $G_{\mathrm{s}}$ represents the conductance of the object, which should be measured by the conductivity sensor. The components $R_{1} \sim R_{4}$ and $C_{1} \sim C_{4}$ model the electrical behavior of the electrode-object interface. The potentials $E_{1} \sim E_{4}$ represent the contact potentials. The values of these potentials depend on the materials of the electrode and the object. Generally, they are not equal, nor are they stable. The components $G_{\mathrm{S} 1}$ and $G_{\mathrm{S} 2}$ represent the conductance path through the object between the force and sense electrodes of the sensor. To measure the conductance $G_{\mathrm{S}}$ accurately, the influence of these parasitics should be eliminated or significantly reduced.

The effect of the contact potentials on the measurement of the $G_{\mathrm{S}}$ can be eliminated by using an ac excitation signal for the sensor. To eliminate the effect of the impedances $R_{1} \sim R_{4}$, $G_{\mathrm{S} 1}$ and $G_{\mathrm{S} 2}$ as well as the effect of the lead-wire resistance, a four-wire measurement is applied. Fig. 2(a) shows a simplified diagram of the four-wire measurement method. In this measurement, the four-electrode sensor is excited with a constant ac current source $I_{\mathrm{ex}}$. The voltage over the sensor conductance $G_{\mathrm{S}}$ is measured with an electronic circuit with high input-impedance. In this measurement, when the measured conductance $G_{\mathrm{S}}$ is low, the voltage over $G_{\mathrm{S}}$ can be very high due to the use of the constant-current excitation signal. This will result in a measurement, which is out of the linear range. Moreover, the voltage between the electrodes 2 and 3 will exceed the free corroding potential.

To overcome this drawback, the conductance $G_{\mathrm{S}}$ can be measured using a constant ac voltage excitation $V_{\text {ex }}$ [see Fig. 2(b)] [5]. In this circuit, the voltage $V_{\text {Gs }}$ over the conductance is fixed to the value of $V_{\text {ex }}$ by the feedback loop around the amplifiers $\mathrm{A}_{1}$ and $\mathrm{A}_{2}$. In case of ideal amplifiers that have a very high loop gain, a negligible input current, and offset voltage, the voltage 


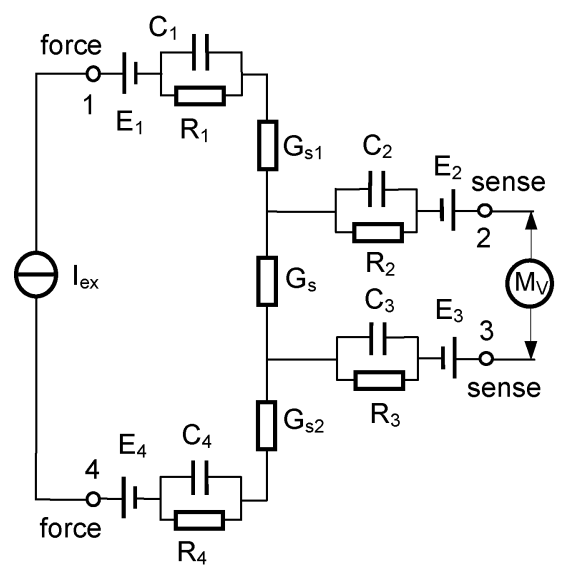

(a)

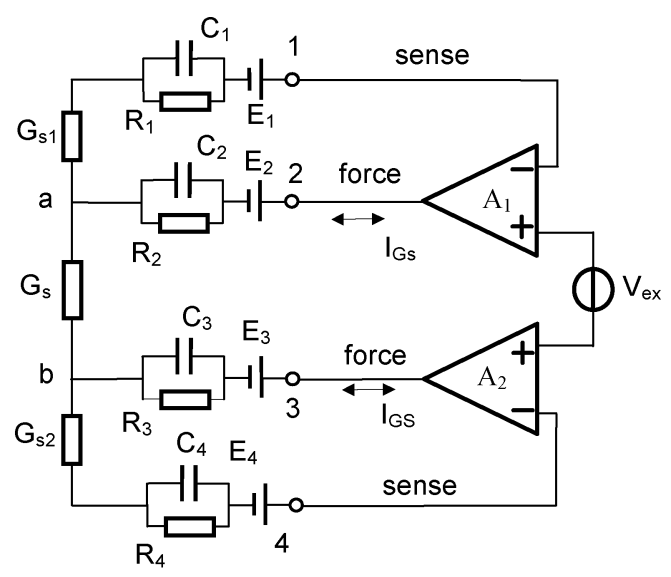

(b)

Fig. 2. Four-wire measurement of the four-electrode conductivity sensor: (a) constant-current excitation and (b) constant-voltage excitation.

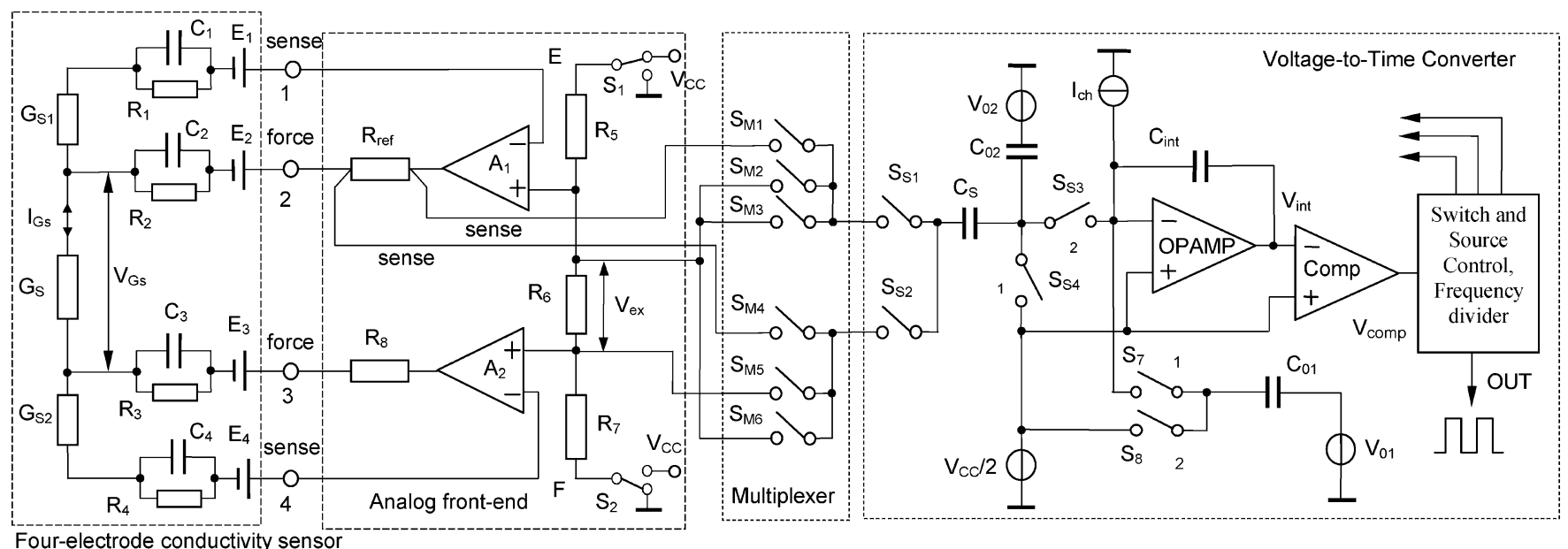

Fig. 3. Simplified interface for the conductance measurement.

$V_{\mathrm{Gs}}$ over the conductance $G_{\mathrm{S}}$ equals $V_{\mathrm{ex}}$. Therefore, the measured conductance equals

$$
G_{\mathrm{S}}=\frac{I_{\mathrm{Gs}}}{V_{\mathrm{Gs}}}=\frac{I_{\mathrm{Gs}}}{V_{\mathrm{ex}}}
$$

So, for a constant voltage $V_{\mathrm{ex}}$, the current flow $I_{\mathrm{Gs}}$ is proportional with the measured conductance.

The described measurement method for the sensor conductance $G_{\mathrm{S}}$ concerns a so-called four-wire method, in which force and sense wires are applied. Such a method reduces the effect of series impedances of the wires and cables that connect the conductivity sensor to the electronic circuit. Meanwhile, the effect of the deposits on the electrode surface of conductivity sensor is reduced as well.

\section{B. Circuit Diagram of the Interface}

Fig. 3 shows a simplified schematic diagram of the interface, which mainly consists of an analog front-end, a multiplexer, and a voltage-to-time converter.

As shown in the above section, in the analog front-end circuit two amplifiers in a unity-gain configuration are employed to establish a controlled excitation voltage $V_{\mathrm{Gs}}$ across the measured conductance $G_{\mathrm{s}}$. The voltage $V_{\mathrm{Gs}}$ is equal to the voltage $V_{\mathrm{ex}}$ across the resistor $R_{6}$. To avoid electrolysis, this voltage should be less than the free corroding potential. The current flow $I_{\mathrm{Gs}}$, which is equal to the current through the resistor $R_{\text {ref }}$ or $R_{8}$, is measured by measuring the voltage $V_{\text {Rref }}$ across the resistor $R_{\text {ref }}$. Both voltages $V_{\text {ex }}$ and $V_{\text {Rref }}$ are directly measured using the voltage-to-time converter via the multiplexer.

The voltage-to-time converter linearly converts the sensor signal (voltage) and the reference signal (voltage) into period-modulated signals by employing a first-order charge-balanced oscillator. The multiplexer, which is formed using six analog switches $\mathrm{S}_{\mathrm{M} 1} \sim \mathrm{S}_{\mathrm{M} 6}$, selects the signal to be measured $V_{\mathrm{ex}}, V_{\mathrm{Rref}}$, or $V_{\mathrm{COM}}$, where $V_{\mathrm{COM}}$ is the offset measurement. The capacitor $C_{\mathrm{s}}$ with two switches $\mathrm{S}_{\mathrm{s} 1}$ and $\mathrm{S}_{\mathrm{s} 2}$ samples one of these three voltages. The charge is dumped into the integrator capacitor $C_{\mathrm{int}}$. The current $I_{c h}$ is periodically integrated and this results in periodic signals.

An ac square-wave excitation signal is generated by using switches $S_{1}$ and $S_{2}$. The use of ac signals is important to reduce electrochemical effects and the effects of dc drift and parasitic Seebeck voltages. Meanwhile, the ac square-wave excitation signal is also used to implement advanced chopping [6], [7], synchronized with voltage-to-time conversion. The chopping technique significantly reduces the effects of offset, $1 / f$ noise, and low-frequency interference. This enables the use of low-cost CMOS technology for accurate measurement systems. 


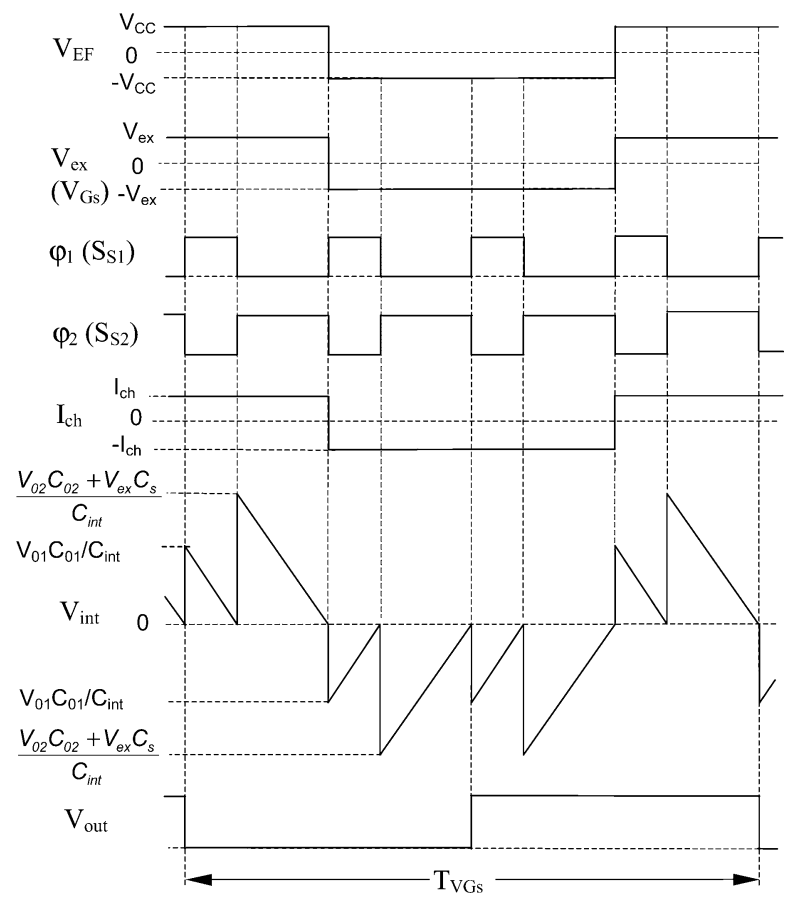

Fig. 4. Some relevant signal levels and control signals in the voltage-to-time converter when the voltage signal $V_{\text {ex }}$ is measured.

Let us suppose that, for example, the voltage $V_{\mathrm{ex}}$ has to be converted into the time domain. Fig. 4 shows some relevant signal levels and control signals in the voltage-to-time converter.

In one complete cycle for the measurement of voltage signal $V_{\text {ex }}$, four measurements are included, which have a chopping sequence of,,$+--++-- \ldots$. The application of such chopping technique eliminates the effect of nonidealities of amplifiers $\mathrm{A}_{1}$ and $A_{2}$ and the effect of the contact potentials $E_{1} \sim E_{4}$, as well as the offset effect of the interface and the effect of any other low-frequency signals.

The use of $C_{01}$ measurement ensures a proper sample-andhold action of the oscillator [6].

As presented in [6] and [7], the periods of the output signal of the oscillator $T_{V G s}, T_{I G s}$, and $T_{o f f}$, corresponding to the measurement of $V_{\mathrm{ex}}, V_{\mathrm{Rref}}$, and $V_{\mathrm{COM}}$, are given by the equations

$$
\begin{aligned}
& T_{V G s}=4 \frac{V_{\mathrm{ex}} C_{\mathrm{s}}}{I_{c h}}+4 \frac{V_{0}\left(C_{01}+C_{02}\right)}{I_{c h}} \\
& T_{I G s}=4 \frac{V_{\mathrm{Rref}} C_{\mathrm{s}}}{I_{c h}}+4 \frac{V_{0}\left(C_{01}+C_{02}\right)}{I_{c h}} \\
& T_{\text {off }}=4 \frac{V_{0}\left(C_{01}+C_{02}\right)}{I_{c h}} .
\end{aligned}
$$

Using these three measurements, the measured result for the conductance of the conductivity sensor is found by the equation

$$
G_{\mathrm{S}}=\frac{T_{I G s}-T_{o f f}}{T_{V G s}-T_{o f f}} \cdot \frac{1}{R_{\mathrm{ref}}}
$$

This result does not depend on the unknown offset and the unknown transfer factor of the interface. In this way, the interface is autocalibrated for additive or multiplicative errors. Even in the case of slow variations of the offset and transfer factor,

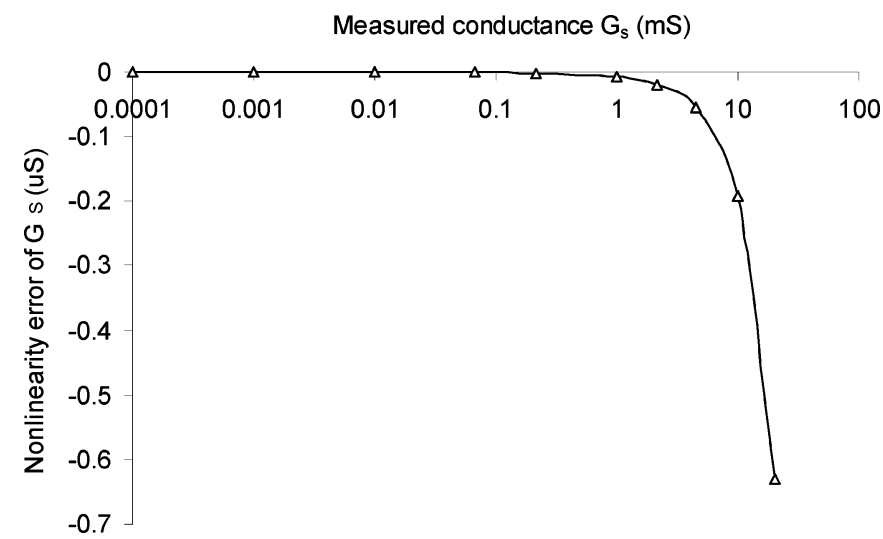

Fig. 5. Nonlinearity errors due to the finite dc gain of amplifiers.

these effects are eliminated. The algorithm can be implemented using, for instance, a microcontroller.

\section{NONIDEALITIES}

As described in Section II, many nonidealities of the interface are eliminated by means of the autocalibration technique [(3)]. However, some effects cannot be eliminated by this technique and should be taken into account during the design of this interface.

\section{A. Finite DC Gain of Op-Amps $\mathrm{A}_{1}$ and $\mathrm{A}_{2}$}

The finite dc gains of the amplifiers $\mathrm{A}_{1}$ and $\mathrm{A}_{2}$ shown in Fig. 3 cause a nonlinearity error in the measurement of conductance $G_{\mathrm{s}}$. When both amplifiers $\mathrm{A}_{1}$ and $\mathrm{A}_{2}$ have a de gain $A_{0}$, the nonlinearity error $\varepsilon_{\text {gain }}$ caused by the finite dc gain is given by

$$
\varepsilon_{\text {gain }}=G_{\mathrm{sm}}-G_{\mathrm{s}}=-\frac{1+\left(R_{8}+R_{\mathrm{ref}}\right) G_{\mathrm{s}}}{1+A_{0}+\left(R_{8}+R_{\mathrm{ref}}\right) G_{\mathrm{s}}} G_{\mathrm{s}}
$$

where $G_{\mathrm{sm}}$ is the measured conductance according to (3) and $G_{\mathrm{S}}$ is the conductance to be measured.

Example: When the dc gain of both amplifiers $\mathrm{A}_{1}$ and $\mathrm{A}_{2}$ is $104 \mathrm{~dB}$ and $R_{\text {ref }}=R_{8}=100 \Omega$, the nonlinearity error due to the finite dc gain of amplifiers is as shown in Fig. 5.

The effect of the contact resistors $R_{2}$ and $R_{3}$ on the linearity is similar to the effect of resistors $R_{\text {ref }}$ and $R_{8}$. To calculate this effect, the resistor values in (4) have to be increased by the values of the corresponding contact resistances

\section{B. Contact Potentials $E_{1} \sim E_{4}$}

Due to the contact potentials $E_{1} \sim E_{4}$ and the finite dc gain of the amplifiers $\mathrm{A}_{1}$ and $\mathrm{A}_{2}$, the voltage $V_{\mathrm{Gs}}$ over the measured conductance amounts to

$$
V_{\mathrm{Gs}}=\frac{A_{0}}{1+A_{0}} V_{\mathrm{ex}}-\frac{A_{0}}{1+A_{0}}\left(E_{1}-E_{4}\right)-\frac{E_{2}-E_{3}}{1+A_{0}} .
$$

It is shown that the contact potentials $E_{1} \sim E_{4}$ cause an additional offset on the voltage over the measured conductance. When the bandwidth of these contact potentials is much less than that of the excitation signal (chopping frequency), this offset will be eliminated by applying the chopping technique as described in the Section II. 


\section{Input Offset Voltage $V_{\mathrm{os}}$ and Biasing Current $I_{\mathrm{bias}}$ of Op-Amps $\mathrm{A}_{1}$ and $\mathrm{A}_{2}$}

The input offset voltages $V_{\mathrm{oS}}$ of the amplifiers $\mathrm{A}_{1}$ and $\mathrm{A}_{2}$ have a similar effect as the contact potentials $E_{1}$ and $E_{4}$. When the bandwidth of the input offset voltages is much less than that of the excitation signal (chopping frequency), their effects will be eliminated by the applied chopping technique.

The biasing currents $I_{\mathrm{Bias} 1}$ and $I_{\mathrm{Bias} 12}$ of the amplifiers $\mathrm{A}_{1}$ and $\mathrm{A}_{2}$ affect not only the measurement of the current $I_{\mathrm{Gs}}$ but, due to the presence of resistive components $R_{1}, R_{4}, G_{\mathrm{S} 1}$, and $G_{\mathrm{S} 2}$, also the measurement of the voltage $V_{\mathrm{Gs}}$. However, these nonidealities cause only additional offsets in the measurements of the current $I_{\mathrm{Gs}}$ and the voltage $V_{\mathrm{Gs}}$, according to the set of equations

$$
\begin{aligned}
& V_{\mathrm{Gs}}=V_{\mathrm{ex}}+I_{\text {Bias } 1}\left(R_{1}-\frac{1}{G_{\mathrm{s} 1}}\right)-I_{\text {Bias } 2}\left(R_{4}-\frac{1}{G_{\mathrm{s} 2}}\right) \\
& I_{\mathrm{Gs}}=\frac{V_{\text {Rref }}}{R_{\text {ref }}}+I_{\text {Bias } 1 .}
\end{aligned}
$$

If the bandwidth of the input biasing currents is much less than that of the excitation signal (chopping frequency), these offsets will be eliminated by the applied chopping technique.

\section{The ON-Resistor $R_{\mathrm{ON}}$ of Sampling Switches}

The ON-resistors $R_{\mathrm{ON}, \mathrm{S}}$ and $R_{\mathrm{ON}, \mathrm{M}}$ of sampling switches $\mathrm{S}_{\mathrm{s} 1}$ or $\mathrm{S}_{\mathrm{s} 2}$ and multiplexer switches $\mathrm{S}_{\mathrm{M} 1} \sim \mathrm{S}_{\mathrm{M} 6}$, together with $R_{5}$, $R_{6}, R_{7}$, and $R_{\text {ref }}$ and the capacitances associated with these resistors, form an RC circuit. For instance, when the voltage $V_{\mathrm{ex}}$ is measured, the time constant of this RC circuit is

$$
\tau_{i}=\left(R_{5} / /\left(R_{7}+R_{6}\right)+R_{\mathrm{ON}, \mathrm{M}}+R_{\mathrm{ON}, \mathrm{S}}\right)\left(C_{\mathrm{s}}+C_{p}\right)
$$

where $C_{p}$ is the parasitic capacitance. This time constant $\tau_{i}$ results in a relative error for the result

$$
\varepsilon_{\mathrm{NL}}=\exp \left(-\frac{T_{p}}{\tau_{i}}\right)
$$

where $T_{p}$ is the minimum time period of the voltage-to-time converter.

Example: When the minimum time period $T_{p}$ is $10 \mu \mathrm{s}, R_{5}=$ $R_{7}=5 \mathrm{k} \Omega, R_{6}=200 \Omega, R_{\mathrm{ON}, \mathrm{M}}=R_{\mathrm{ON}, \mathrm{S}}=1 \mathrm{k} \Omega$, $C_{p}=50 \mathrm{pF}$, and $C_{\mathrm{s}}=28 \mathrm{pF}$, the relative error due to the time constant $\varepsilon_{\mathrm{NL}}$ amounts to $3 \times 10^{-9}$.

\section{E. Temperature Drift}

As described in Section II, the offset and gain errors of the interface are eliminated by applying the autocalibration (3). This autocalibration will also eliminate the drift of the offset and gain of the interface due to the temperature variations. Yet, the reference resistor $R_{\text {ref }}$ should have a low temperature coefficient because its temperature performance will directly affect the absolute measurement result of the conductance $G_{\mathrm{S}}$ [see (3)].

\section{EXPERIMENTAL RESULTS}

The proposed sensor interface has been implemented and tested using the circuit shown in Fig. 3. For the operational amplifiers, a dual op-amp (OPA2132PA) has been

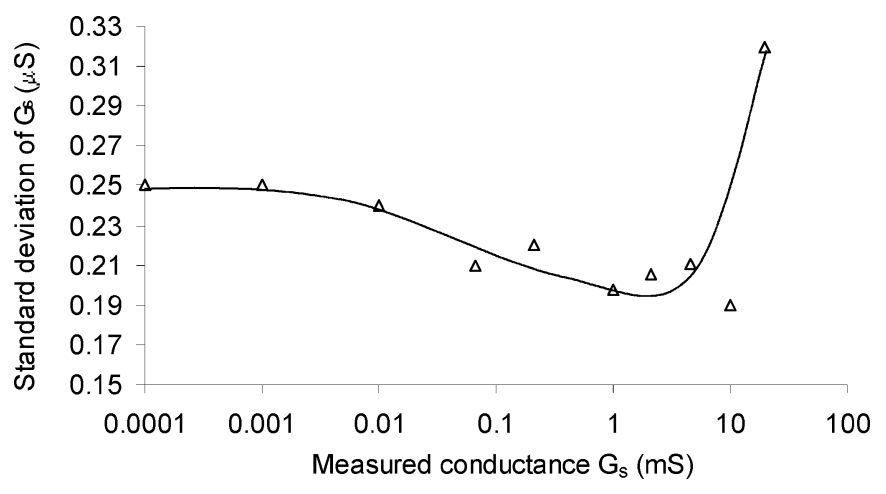

Fig. 6. The standard deviation of the random error of the conductance measurement for a measurement time of $110 \mathrm{~ms}$.

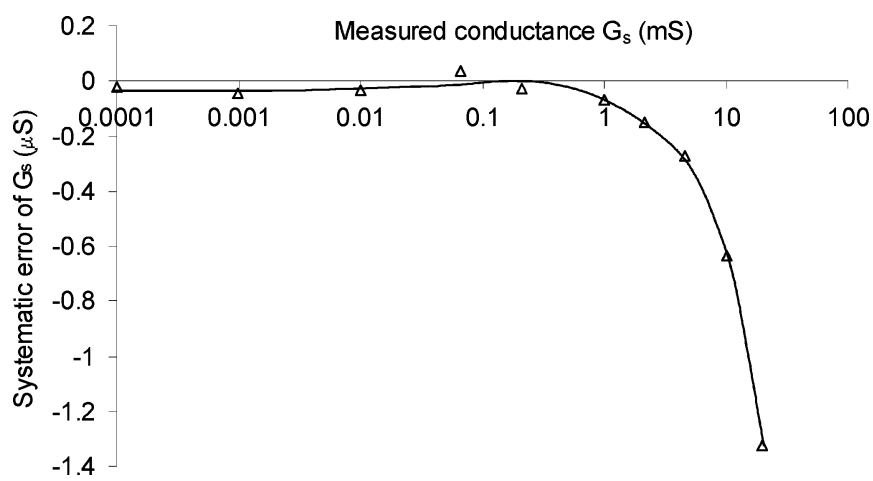

Fig. 7. Systematic error of the interface. Each of the plotted data points represents the average of 100 measurement results.

used. The voltage-to-time converter and multiplexer have been implemented with a universal transducer-interface chip (UTI03-A79C), which has been realized using low-cost CMOS technology [8]. The ac square-wave excitation signal is generated by the voltage-to-time converter itself. The frequency of the excitation signal amounts to $7 \mathrm{kHz} \sim 12.5 \mathrm{kHz}$ depending on the value of measured conductance. A microcontroller of the type PIC16F876, which has a $5 \mathrm{MHz}$ counting frequency, is employed to measure the output period of the voltage-to-time converter. It also processes the data and communicates with the outside digital world. The system is powered by a single $5 \mathrm{~V}$ supply voltage.

The performances of the interface have been tested for the case that $R_{\text {ref }}=100.076 \Omega$ with a temperature coefficient of $10^{-6} / \mathrm{K}$ and $G_{\mathrm{S}}=0.1 \mu \mathrm{S}$ to $20 \mathrm{mS}$, with a measurement time of about $110 \mathrm{~ms}$. The controlled excitation voltage $V_{\text {ex }}$ has been applied with a peak-to-peak value of $200 \mathrm{mV}$. Depending on the electrochemical properties, the amplitude of the excitation signal can be adjusted for a value less than the free corroding potential. The main measurement results for the conductance $G_{\mathrm{s}}$ are depicted in Figs. 6 and 7, which show the standard deviation of the random error and the systematic error, respectively. The standard deviation has been derived for a large number of measurements, each with a measurement time of $110 \mathrm{~ms}$. When determining the systematic error (Fig. 7), the effect of the random error has been reduced by taking the average of 100 measurements for each data point plotted in the figure. 
It is shown that the interface has a standard deviation of $1.6 \times 10^{-5}(0.32 \mu \mathrm{S})$ and a systematic error of $6.6 \times 10^{-5}$ $(1.32 \mu \mathrm{S})$ over a conductance range of $0.1 \mu \mathrm{S}$ to $20 \mathrm{mS}$ and a measurement time of about $110 \mathrm{~ms}$. Two successive measurements over a conductance range of $0.1 \mu \mathrm{S}$ to $20 \mathrm{mS}$ have been performed, which show that the repeatability of the interface amounts to $0.18 \mu \mathrm{S}$.

Fig. 7 shows that the systematic error increases with increasing conductance $\mathrm{G}_{\mathrm{s}}$. As discussed in Section III, this is due to the finite dc gain of the amplifier $\mathrm{A}_{1}$ and $\mathrm{A}_{2}$.

\section{CONCLUSIONS}

In this paper, an interface for conductivity sensors has been presented. A low-cost interface with a high accuracy and good long-term stability has been realized by applying advanced measurement techniques including four-wire measurement technique, ac square-wave excitation, chopping and autocalibration. The controllable excitation signal for conductivity sensors enables avoiding the electrolysis. Tests have been performed over the conductance range of $0.1 \mu \mathrm{S}$ to $20 \mathrm{mS}$. It is shown that, with a measurement time of about $110 \mathrm{~ms}$, the interface can measure the conductance with a standard deviation of $1.6 \times 10^{-5}$ and a systematic error of $6.6 \times 10^{-5}$. The proposed interface is very suitable for implementation in low-cost CMOS technology.

\section{REFERENCES}

[1] Product Guide for Process Measurement Instrumentation, Thornton Inc., 2000.

[2] Specification Data Sheets, Falmouth Scientific, Inc., 2000.

[3] A. J. Fougere, N. L. Brown, and E. Hohart, "Integrated CTD oceanographic data collection platform," in Oceanology 92, Brighton, U.K., 1992.

[4] Model 4081 Conductivity Meter, Amber Science Inc., 1999.

[5] T. R. Barben, "Four electrode conductivity sensor," U.S. Patent 641 254, Oct. 1978.
[6] F. van der Goes, "Low-cost smart sensor interfacing," Ph.D. dissertation, Delft Univ. of Technology, 1996.

[7] F. van der Goes and G. C. M. Meijer, "A novel low-cost capacitivesensor interface," IEEE Trans. Instrum. Meas., vol. 45, pp. 536-540, Apr. 1996.

[8] Users Guide for Universal Transducer Interface (UTI), Revolution in Sensor Interfacing, Smartec BV, The Netherlands, 1997.

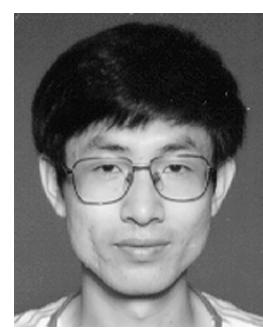

Xiujun Li (SM'03) was born in Tianjin, China, on February 19, 1963. He received the B.Sc. degree in physics in 1983, the M.Sc. degree in electrical engineering from Nankai University, Tianjin, in 1986, and the Ph.D. degree from the Department of Electrical Engineering, Delft University of Technology, Delft, The Netherlands, in 1997.

From 1996, he was a Senior Researcher with the Faculty of Information Technology and Systems, Delft University of Technology, where he was involved in research and the development of smart capacitive sensors and low-cost high-performance interfaces for smart sensors. Since August 2001, he has been with Bradford Engineering B.V., working on sensors and sensor systems for the space application. His research interests are smart sensors, smart sensor systems, and smart signal processing.

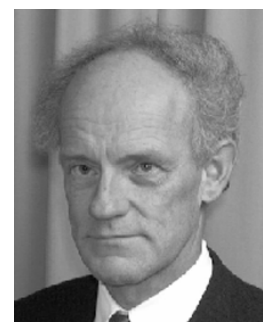

Gerard C. M. Meijer (SM'99) was born in Wateringen, The Netherlands, on June 28,1945 . He received the Ingenieurs (M.S.) and Ph.D. degrees in electrical engineering from the Delft University of Technology, Delft, The Netherlands, in 1972 and 1982, respectively.

Since 1972, he has been with the Laboratory of Electronics, Delft University of Technology, where he is an Associate Professor, engaged in research and teaching on analog ICs. In 1984 and part-time during 1985-1987, he was involved in the development of industrial level gauges and temperature transducers. In 1996, he was a Founder of Sensart, where he is a Consultant in the field of sensor systems.

Dr. Meijer is a member of the Netherlands Society for Radio and Electronics. 